\title{
Combined search of MeV $v$ and gravitational waves from astrophysical impulsive sources
}

\author{
Giulia Pagliaroli*, Odysse Halim \\ Gran Sasso Science Institute, Viale Francesco Crispi 7, I-67100 L'Aquila, Italy \\ INFN-LNGS, I-67100 L'Aquila, Italy \\ E-mail: giulia.pagliaroliegssi.it \\ odysse.halimegsi.it
}

\section{Claudio Casentini, Viviana Fafone}

Universitá di Roma Tor Vergata, via della Ricerca Scientifica, I-00133 Roma, Italy

INFN Sezione di Roma Tor Vergata, via della Ricerca Scientifica, I-00133 Roma, Italy

E-mail: claudio.casentiniegmail.com

viviana.fafonedroma2.infn.it

\section{Carlo Vigorito}

Universitá di Torino \& INFN, via Pietro Giuria 1, I-10125 Torino, Italy

E-mail:vigoritodto.infn.it

\begin{abstract}
The search of astrophysical impulsive sources emitting low-energy neutrinos and Gravitational Waves (GWs) can be challenging for different reasons: weak signals at the source, large distance of the source, high background inside the detectors. A joint search in a network of GWs and $v$ detectors can provide several benefits: temporal coincidences of GWs and $\mathrm{MeV} v$ bursts improve sensitivity and confidence of detection and a correlation studies of the two probes provide complementary informations on the physical mechanisms at work inside the source. In this paper we summarise the strategy for such a combined search defined during the last years. Moreover, we apply, for the first time in this context, a novel method that increases the probability of identifying impulsive astrophysical bursts of low-energy neutrinos. The proposed approach exploits the temporal structure differences between astrophysical bursts and background fluctuations and it allows us to pinpoint weak signals otherwise unlikely to be detected.
\end{abstract}

Neutrino Oscillation Workshop (NOW2018)

9 - 16 September, 2018

Rosa Marina (Ostuni, Brindisi, Italy)

${ }^{*}$ Speaker. 


\section{The strategy for a combined GWs- $v$ search}

Several known astrophysical sources are expected to emit both gravitational waves and lowenergy neutrinos. In this work, we consider transient sources, causing both a GWs burst $(\sim 10 \mathrm{~ms})$ and an impulsive ( $\sim 10$ seconds) emission of low-energy, $(\sim 10 \mathrm{MeV})$, neutrinos. In particular, we are focused on Core-Collapse SNe (CCSNe) [1], "Failed" SNe [2], and Quark Novae [3].

Despite the large amount of total energy $\left(\sim 10^{53} \mathrm{ergs}\right)$ released in neutrinos, when the source distance increases and/or the average energy of emitted neutrinos decreases the signal statistics drops and the identification of these low statistics astrophysical bursts could be challenging. The situation for the GWs detection is also more complex, due to the large uncertainty on the expected amplitude of the signals [4]. Moreover, burst signals easily mimic the (non-stationary) part of the noise of the interferometers (the so-called glitches) and a search based on reliable templates cannot be performed due to the stochastic nature of the emission processes.

In order to overcome these detection problems a combined search among GWs interferometers and $v$ detectors has been proposed [5]. Indeed, gravitational wave and neutrino signals are expected to be emitted and detected within a tight window, ranging from a few milliseconds to a few seconds, depending on the dominant GW emission process [6]. This justify the search for temporal coincidences between $\mathrm{GW}$ and $v$ within the conservative window $w_{c}=20$ seconds. The detectors involved up to now are the GWs interferometers, Advanced LIGO and Advanced Virgo [11], and the neutrino detectors: Borexino [7], Large Volume Detector (LVD) [12], Kamland [8] and IceCube [9].

The combined False Alarm Rate (FAR) for the global network of detectors is defined as

$$
F A R=R_{G W} \cdot R_{v} \cdot 2 w_{c},
$$

where $R_{G W}\left(R_{v}\right)$ is the rate of background coincidences in the GWs $(v)$ subnetwork, respectively. The factor two accounts for the fact that coincidence searches generally impose the relative timing of a GW and neutrino event to be within $w_{c}$, but not in a specific order. For our analysis we fixed the global $\mathrm{FAR}=1 / 1000$ years. The adopted strategy is to consider as a first step the two sub-networks of GWs and $v$ detectors as independent. Once GW burst candidates and $v$ burst candidates are defined in the respective subnetwork, the final list of candidate astrophysical events is obtained looking for GW- $v$ coincidences within $w_{c}$.

The search of GW bursts is performed by using the cWB pipeline[10] designed to the search for un-modelled bursts. In this pipeline coincident events are ranked according to their coherent network signal-to-noise ratio (SNR) $\eta_{c}$, favoring GW signals correlated in the detectors and suppressing uncorrelated glitches. So that, the accidental background rate of GW coincidences in the previous Eq.(1.1), is expressed as a function of this statistical parameter, i.e. $R_{G W}\left(\eta_{c}\right)$. Real temporal coincidences among interferometers, called 0-lag coincidences, are compared with accidental ones estimated using the time-slide background distribution and this comparison provides their significances or false alarm rates [11].

While for the GW sub-network the definition of the burst candidates and of their significances is discussed and well defined in previous literature, for the neutrino sub-network this work is missing. With the aim to define a coherent network SNR $\eta_{v}$, also for the $v$ sector we discuss in the following a new approach for the search of neutrino bursts. 


\section{The new method for the search of astrophysical $v$ bursts}

In all the considered $v$ detectors the search of astrophysical bursts is based on the definition of clusters as the group of the events contained in consecutive time windows of $w_{c}$. Each cluster is characterised by its multiplicity $m_{i}$ and its time duration $\Delta t_{i}$, defined as the time difference between the first and the last event detected inside the window. In order to increase the detection probability this search is performed one more time by shifting the consecutive time windows of 10 seconds with respect to the first search. Standard search procedures assume a selective cut on the imitation frequency of the cluster $f_{i}^{i m}$ (or equivalently on $m_{i}$ ) in order to reduce the background [12]. This background reduction is purely statistical and no physical characteristics of the signal are used in order to separate background clusters from signal clusters.

As demonstrated [13] this search method can be improved by exploiting the temporal structure differences between astrophysical bursts and background fluctuations. For each cluster of events we define the parameter, $\xi_{i}=\frac{m_{i}}{\Delta t_{i}}$ as the ratio between the cluster multiplicity and the cluster duration. Impulsive sources are expected to produce clusters of neutrino events very short in time with respect to the time window duration, i.e. high values of $\xi_{i}$. On the other hand, the local background is expected to be flat over the time window without peculiar temporal structure, i.e. $\delta t_{i} \simeq w_{c}$ and, so that, very small values for $\xi_{i}$. We consider only event clusters with $m_{i} \geq 2$. We simulate 10 years of background data for the neutrino detectors and we perform also a Monte Carlo extraction of the astrophysical clusters expected in each neutrino detector by considering different source distances $D \in(8.5-500) \mathrm{kpc}$ and by assuming basic temporal and spectral shapes, details are in Ref. [13]. Simulated signals are randomly injected inside the simulated background. The Probability Density

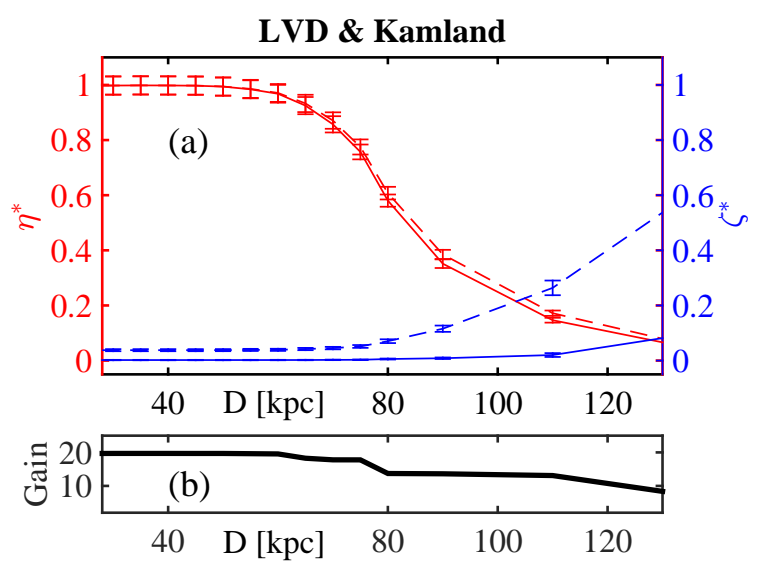

Figure 1: Panel (a): Red lines show the detection efficiencies curves $\eta^{*}$ whereas blue lines show the misidentification probability curves $\zeta^{*}$ for the network LVD \& Kamland. Solid (Dashed) lines are obtained by following the new (standard) method for background reduction. Panel (b): The gain factor for the network LVD \& Kamland as defined in the text as a function of the source distance D.

Distribution (PDF) of pure background clusters as functions of the $\xi$ parameter is characterised by very small values of $\xi$, whereas clusters where an astrophysical signal is present show a PDF shifted at higher values of $\xi$, cumulating faster in time $\Delta t_{i}<w_{c}$. So that we can define an optimal value of the cut parameter $\bar{\xi}_{X}$ allowing a clear separation of signals from backgrounds. The optimal cut parameters found are reported in Tab.1 of Ref. [13]. 
For detectors working as a network we require that the product of the $\xi_{X}$ of coincident clusters is greater than the global cut value $\bar{\xi}^{*}=\sqrt[N e t]{\prod_{X=1}^{N e t} \bar{\xi}_{X}}$. The sensitivity of the neutrino network can be obtained by using the following definition of detection efficiency $\eta^{*}$, i.e. the number of astrophysical clusters surviving the statistical cut on $f^{i m}$ that are found in temporal coincidence and are also characterised by a global $\bar{\xi}^{*}$ greater than the cut value defined above over the total injected signals. In a similar manner the network definition of the misidentification probability $\zeta^{*}$ is the ratio among background coincidences and the total number of 0-lag coincidences found.

As a leading example we show the joint analysis of LVD and Kamland. The detection efficiency and the misidentification probability of this sub-network are plotted in Panel (a) of Fig.1. Dashed lines represent the old method based on statistical cut plus temporal coincidence search, whereas solid lines show the same quantities obtained by adding the $\bar{\xi}^{*}$ cut described above. We also show the gain factor, $G=\zeta^{* \prime} / \zeta^{*}$, calculated as the ratio between the misidentification probability before $\left(\zeta^{* \prime}\right)$ and $\operatorname{after}\left(\zeta^{*}\right)$ the $\bar{\xi}^{*}$ cut.

\section{Summary}

We discussed the strategy for a combined search of astrophysical impulsive sources of GWs and $\mathrm{MeV}$ neutrinos. In this context we incorporate a new method for the search of low energy neutrino bursts that can decrease the misidentification probability of a factor 10-20 without losing detection efficiency, as reported in the Panel (b) of Fig.1. The proposed method applied to the neutrino sub-network provides an increase of its detection horizon. Moreover, the introduction of the $\xi$ parameter is the first step versus the definition of a suitable coherent statistical description of the signal to noise ratio fo the neutrino sector.

\section{References}

[1] H. T. Janka, et al., Phys. Rept. 442 (2007) 38.

[2] S. M. Adams, et al., Mon. Not. Roy. Astron. Soc. 469 (2017) 1445.

[3] R. Ouyed, J. Dey and M. Dey, Astron. Astrophys. 390 (2002) L39.

[4] C. Ott, Class. Quant. Grav. 26 (2009) 063001.

[5] I. Leonor, et al., Class. Quant. Grav. 27 (2010) 084019.

[6] G. Pagliaroli, F. Vissani, E. Coccia and W. Fulgione, Phys. Rev. Lett. 103 (2009) 031102.

[7] G. Alimonti, et al. [Borexino Collaboration], Nucl. Instrum. Meth. A 600 (2009) 568.

[8] K. Eguchi, et al. [KamLAND Collaboration], Phys. Rev. Lett. 90 (2003) 021802.

[9] L. KŽpke [IceCube Collaboration], J. Phys. Conf. Ser. 1029 (2018), no. 1, 012001.

[10] Klimenko, et al., Class. Quant. Grav. 25 (2008) 114029.

[11] B. P. Abbott, et al. [LIGO Scientific and Virgo Collaborations], Phys. Rev. D 95 (2017) no.4, 042003.

[12] N. Y. Agafonova, et al. [LVD Collaboration], Astrophys. J. 802 (2015) no.1, 47.

[13] C. Casentini, G. Pagliaroli, C. Vigorito and V. Fafone, JCAP 1808 (2018) no.08, 010. 